\title{
Giant Fibrovascular Polyp of the Larynx
}

\author{
Silva Bona do Nascimento ${ }^{1}$ Flávio Carvalho Santos ${ }^{2}$ Mariana de Novaes Santos Magalhães Pinheiro ${ }^{3}$ \\ 1 Department of ENT, Instituto de Neurociencias, Teresina, Piaui, Brazil \\ 2 Department of Otolaryngology, Hospital Flávio Santos, Teresina, \\ Address for correspondence Silvia Bona do Nascimento, MD, MSc, \\ Piaui, Brazil \\ ${ }^{3}$ Santa Casa de Misericórdia, São Paulo, São Paulo, Brazil \\ Department of Otorinolaringologia, Instituto de Neurociencias, Rua \\ Bartolomeu Vasconcelos 2440 Bairro Ilhotas, Teresina, Piaui \\ 64049570, Brazil (e-mail: silviabona@yahoo.com).
}

Int Arch Otorhinolaryngol 2014;18:325-327.

\begin{abstract}
Keywords

- larynx

- polyps

- airway obstruction

Introduction Fibrovascular polyps (FVPs) are rare benign tumors originating in the cervical esophagus, and, rarely, in the hypopharynx. A case of FVP from the larynx has not yet been described in the literature.

Objectives To discuss a unique case of FVP originating in the larynx.

Resumed Report A 58-year-old woman presented with a 6-month history of progressive dysphagia. Endoscopic exams identified an elongated polypoid lesion originating from the mucosa of the epiglottis and the right pharyngoepiglottic fold. Excision of this mass under direct laryngoscopy revealed an $11.5 \times 0.8-\mathrm{cm}$ polypoid lesion, histologically diagnosed as an FVP.

Conclusion The location of this FVP is important because, despite being a benign tumor, it carries a potentially lethal risk of upper airway obstruction.
\end{abstract}

\section{Introduction}

Fibrovascular polyps (FVPs) are rare benign submucosal tumorlike lesions originating in the cervical esophagus, and, rarely, in the hypopharynx. ${ }^{1,2}$ Their importance is due to the potential risk of regurgitation into the airway and asphyxia. We describe the case of a giant FVP originating from the epiglottis and the pharyngoepiglottic fold, unmentioned in the medical literature.

This project was approved by the Hospital Flavio Santos Ethics Committee under protocol number 01/2013.

\section{Case Report}

A 58-year-old woman presented with a 6-month history of progressive dysphagia characterized by a strange body sensation in the hypopharynx. The patient distinctly referred that the exact location of the mass varied throughout time, mainly during swallowing. On two occasions, the patient felt that she regurgitated an elongated mass into the oral cavity. During these episodes, the patient felt she was suffocating, obtaining quick relief when she swallowed the mass. Besides these, there were no other otolaryngologic symptoms. Due to the dysphagia, the

received

October 30, 2013

accepted

November 24, 2013

published online

May 13, 2014
DOI http://dx.doi.org/

10.1055/s-0033-1364171. ISSN $1809-9777$. patient had lost $5 \mathrm{~kg}$. During physical examination, no alterations were observed. Initially, the patient was submitted to an endoscopic larynx examination, which failed to show any anomalies. Further diagnostic evaluation with upper digestive endoscopy identified a sausage-shaped polypoid lesion in the esophageal lumen that, when pulled, could be seen on the outside of the oral commissure ( - Fig. 1). A second videolaryngoscopy identified a pedicle originating from the mucosa of the epiglottis and the right pharyngoepiglottic fold ( - Fig. 2). Excision of this mass was performed under general anesthesia. Initially, the mass was completely exposed by direct laryngoscopy. The pedicle was gently cauterized and cut. Macroscopically, the mass was a polypoid lesion of $11.5 \times 0.8 \mathrm{~cm}$, with a distal extremity covered by desquamation and thickening of the epithelium ( - Fig. 3 ). The anatomopathologic exam showed a discreetly fibrotic stroma with abundant venous and arterial vascularization immerse in a mainly mononuclear inflammatory infiltrate, characterizing an FVP.

\section{Review of Literature with Differential Diagnosis}

FVPs of the airway are diagnostic challenges. Identification of the lesion is much easier when the patient is able to

Copyright $\odot 2014$ by Thieme Publicações License terms Ltda, Rio de Janeiro, Brazil

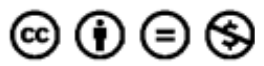




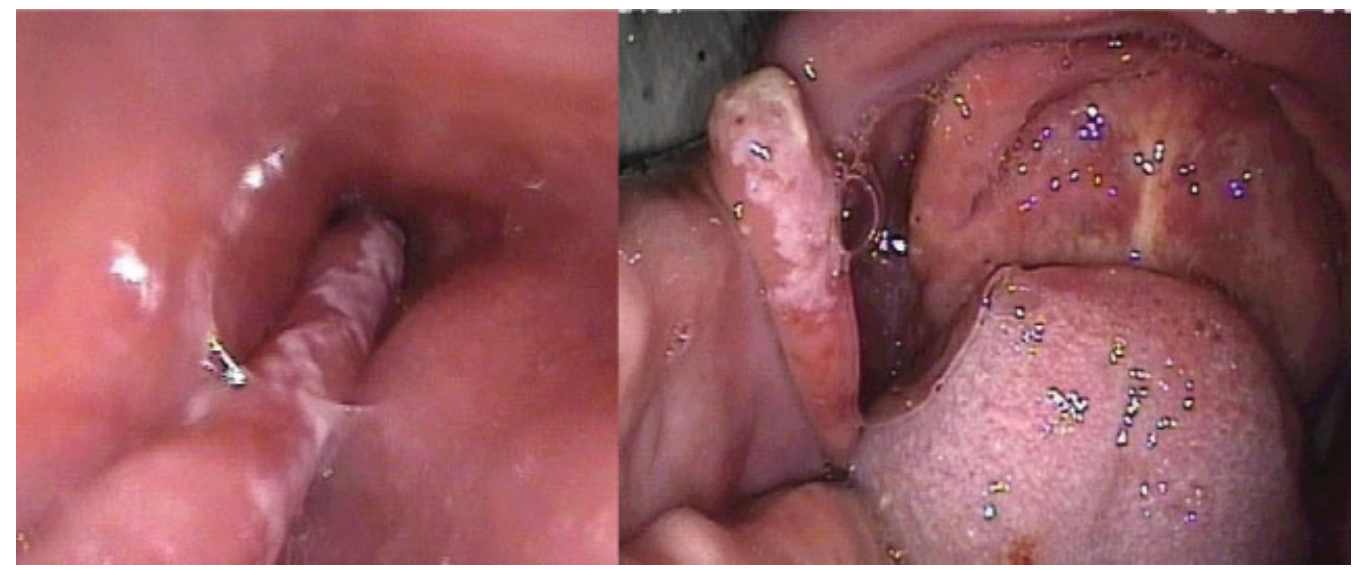

Fig. 1 Endoscopic view of the polyp in the esophagus and protruding through the mouth.

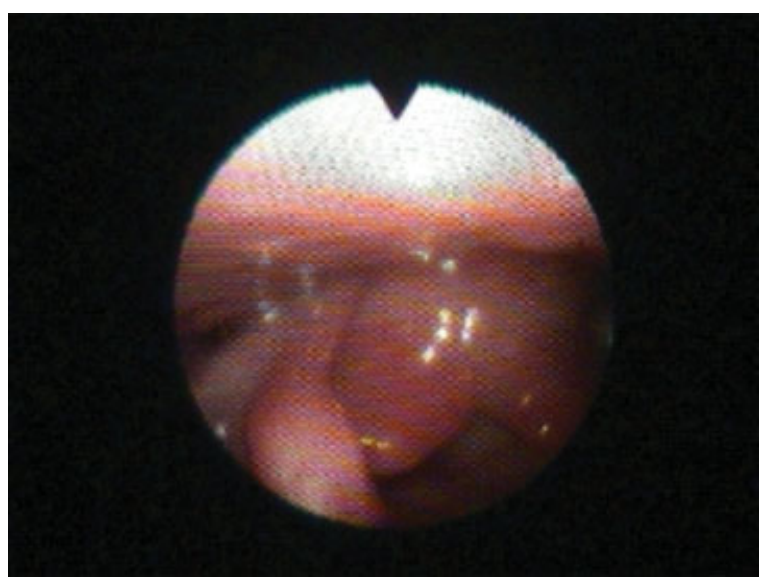

Fig. 2 Endoscopic view of the polyp's origin from the epiglottis and right pharyngoepiglottic fold.

regurgitate it into the oral cavity. ${ }^{3}$ When similar lesions arise from the esophagus, up to $25 \%$ are undetected on endoscopic examination. ${ }^{4}$ This happens because these lesions are typically very similar to the normal hypopharyngeal and esophageal mucosa. ${ }^{5}$ On - Fig. 2, it can be noted that during laryngoscopic examination, detection of the lesion is possible only after meticulous evaluation of the right pharyngoepiglottic fold.

Regarding other diagnostic exams, a barium swallow normally reveals an esophageal lumen defect, but often fails to identify its origin, absolutely necessary for surgical planning. ${ }^{3}$

Because of the limitations of the endoscopic and radiographic exams, the gold standard for the diagnosis of intraluminal lesions of the esophagus and hypopharynx are computed tomography (CT) scans and magnetic resonance imaging (MRI). Even when the diagnosis has been previously established by other methods, in larger lesions, CT scans of the neck and thorax are necessary to establish the limits and size of the lesion, its relation to the esophagus, and the origin of the stalk, all factors that define the optimal surgical technique for resection. ${ }^{3}$ MRI is superior to $\mathrm{CT}$ when vascularization must be assessed. ${ }^{5}$

The differential diagnosis of FVPs includes other conditions that produce similar symptoms, such as acalasia, pharyngeal or esophageal compression by extrinsic lesions or other intraluminal polypoid lesions (lipomas, inflammatory polyps, hemangiomas, lymphangiomas, schwannomas, or leiomyosarcomas, as well as other rare tumors such as carcinoid tumors or paragangliomas). ${ }^{3,5}$ However, on imaging studies, FVPs appear as typical smooth and elongated sausage-shaped masses, with discreetly bullous tips, well represented on - Fig. 3.

Obviously, a definitive diagnosis is accomplished only by histopathologic evaluation. Histologically, FVPs present a mixture of fibrous elements, adipose tissue, and blood vessels, surrounded by normal squamous epithelium. ${ }^{5}$

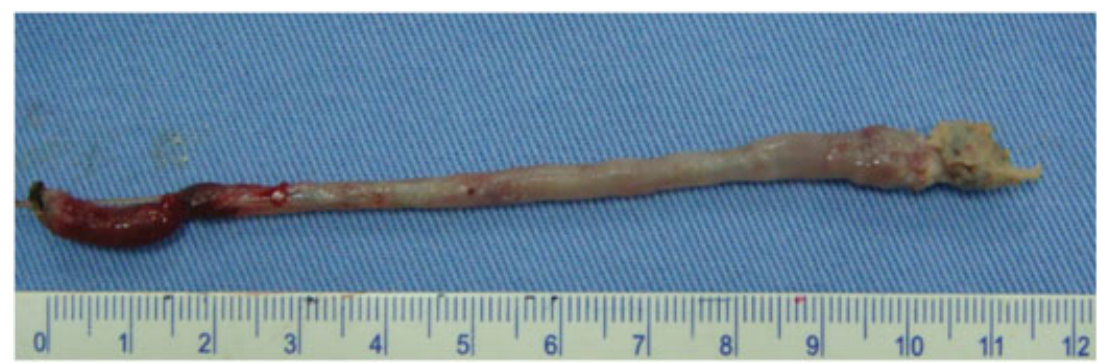

Fig. 3 Macroscopic view of the polyp. 


\section{Discussion}

FVPs are rare benign tumors, with a few more than 100 cases reported in the literature today, ${ }^{3}$ usually originating in the cervical esophagus near the upper esophageal sphincter. ${ }^{1}$ FVPs originating in the hypopharynx are even more unusual, with only a few cases described up to now. ${ }^{4}$ In this case, the polyp originated from the epiglottis and the right pharyngoepiglottic fold. A similar case is unheard of in the medical literature. Therefore, the FVP presented here is a very rare variant of the classic FVPs emerging from the esophagus or hypopharynx.

The exact mechanism of formation of a FVP is not known; however, most hypothesis sustain that it is directly associated to the forces that act on the esophageal and pharyngeal mucosa during swallowing. When the lesion extends inside the esophagus, esophageal peristalsis also contributes to its growth. ${ }^{4,5}$ Furthermore, the nonexistence of muscular support and the flaccid mucosa in these areas are predisposing conditions for FVPs. ${ }^{5}$

FVPs are commonly known to arise from two locations: the area between the superior and inferior cricopharyngeal muscles (the Killian triangle) or between the inferior cricopharyngeal muscle and the proximal esophagus (the Laimer triangle). ${ }^{4}$ However, the polyp presented here was from neither of these locations.

FVPs of the pharynx and esophagus are more prevalent in middle-aged and elderly males; however, cases have been reported in patients of all ages and of both genders, even in infants. $^{3}$

The symptoms of an FVP vary mainly according to its size and location. Being more common in the cervical esophagus, it is possible they produce no symptoms and stay undetected for several years. ${ }^{6}$ When symptoms are present, however, the most common are dysphagia (87\%), respiratory symptoms (cough, dyspnea, and stridor in 25\%), and regurgitation of a fleshy mass into the mouth $(12 \%)^{6}$

In this case, the patient had a typical symptom of suffocation when the mass protruded into the oral cavity. However, swallowing of the mass relieved this symptom, because then the FVP returned to the esophagus. In has been previously reported that patients with similar conditions presented with acute upper airway obstruction. ${ }^{5}$ Therefore, it must be em- phasized that, regardless of being benign lesions, the FVPs of the hypopharynx carry a risk of glottic and acute upper airway obstruction. Furthermore, it is associated with bleeding and anemia, malignant transformation, and chronic emaciation because of the dysphagia. ${ }^{6}$

Treatment consists of surgical excision of the polyp. The surgical approach must be carefully chosen after analysis of the origin of the stalk, vascularization, and size of the polyp. ${ }^{5}$ Traditionally, lesions larger than $8 \mathrm{~cm}$ in length are removed by cervicotomy. ${ }^{5}$ However, in this case, the tumor's small diameter allowed its complete endoscopic resection. Surgical excision was accomplished by gentle pulling of the pedicle, followed by electrocauterization of its base. Surgical removal of this kind of lesion usually ensures no recurrences, but some have been described in the literature. ${ }^{6}$

If the patient presents in an emergency setting with upper airway obstruction, tracheostomy may be necessary to establish an airway prior to definitive surgical resection. ${ }^{4}$

\section{Conclusion}

The existence of a giant FVP arising from the supraglottis is important because despite being a benign lesion, it encompasses a potentially lethal risk of upper airway obstruction. This case report shines attention on this condition, which requires a high degree of clinical awareness to be diagnosed.

\section{References}

1 Zevallos JP, Shah RP, Baredes S. Giant fibrovascular polyp of the hypopharynx. Laryngoscope 2005;115(5):876-878

2 Al-Swiahb JN, Al-Dousary SH. Fibrovascular polyp of the hypopharynx. Ann Saudi Med 2008;28(3):217-219

3 Madeira FP, Justo JWR, Wietzycoski CR, Burttet LM, Kruel CDP, da Rosa AP. Giant fibrovascular polyp of the esophagus: a diagnostic challenge. Arq Bras Cir Dig 2013;26(1):71-73

4 Ozdemir S, Gorgulu O, Selcuk T, Akbas Y, Sayar C, Sayar H. Giant fibrovascular polyp of the hypopharynx: per-oral endoscopic removal. J Laryngol Otol 2011;125(10):1087-1090

5 I H, Kim JS, Shim YM. Giant fibrovascular polyp of the hypopharynx: surgical treatment with the biappoach. J Korean Med Sci 2006;21(4):749-751

6 Lee SY, Chan WH, Sivanandan R, Lim DTH, Wong WK. Recurrent giant fibrovascular polyp of the esophagus. World J Gastroenterol 2009;15(29):3697-3700 